\title{
Research on the Solar Energy Biaxial Automatic Tracking System Based on the GPS and GPRS
}

\author{
Yang Bai \\ School of North China Electric Power University, Baoding 071000, China; \\ 838264478@qq.com
}

Keywords: GPS, GPRS, Solar Energy, Sun Trajectory Tracking, the Photoelectric Tracking.

\begin{abstract}
With the continuous consumption of resources, the demands for energy and the consumption of non-renewable energy is growing more and more. Solar energy, as a green initiative non-polluting renewable energy, has a great prospect. But most conventional solar panels are fixed, causing the exploitation insufficient. This paper will put forward another solar energy biaxial automatic tracking system based on the GPS and GPRS module. This paper introduces the designs on the software and hardware construction of the system, and shows the hardware circuit design of the system.
\end{abstract}

\section{Introduction}

Nowadays, energy has become the most basic power of economic development. Besides, the larger population and the faster the development of the economy are, the more obvious the trend is. At present, our main energy sources are coal, oil and natural gas, which are non-renewable resources. The non-renewable resources will finally diminish with the consumption. Solar energy, as a new regenerative resource raised people's attention. Compared with conventional energy, solar energy has the advantages of pollution-free, widely distributed and rich reserves. It is an ideal substitute of traditional energy[1]. But the conversion efficiency of existing devices is low. To improve the efficiency is what people have been studying.

Firstly, this paper introduces the design of hardware mechanism structure. The mechanism is divided into two parts-the upper swing mechanism controlling east-west axis and the lower swing mechanism controlling north-south axis. Secondly, by comparing two conventional solar panels control strategies, an advanced control scheme based on the GPS and GPRS modules is put forward. The principle of it to combine the sun trajectory tracking and webservice. In the final of this paper the hardware circuit design is shown.

\section{Mechanical Structure Design.}

This paper adopts the structure of north-south axis - east-west axis solar tracking device based on dual axis, which is composed of an upper swing module and a lower swing module tracking east-west and north-south respectively, in order to realize the solar panels altitude and azimuth angle tracking[2][3].

The dual axis tracking mechanical structure selects north-south - east-west axis as the spindle. The structure adopts modular design to simplify the design, manufacture and install, containing east-west set upper swing module and north-south set lower swing muddle.

The actuating mechanism is composed of solar panels, panels-supporting part, upper swing mechanism and hem rotating mechanism. The upper swing mechanism is composed of an upper drive shaft, an upper electronic rotary table, an upper swing supporting pedestal; and it is installed in east-west direction. Similarly the lower swing mechanism is composed of an lower drive shaft, an lower electronic rotary table, an lower swing supporting pedestal; and it is installed in north-south direction. And there are two step motors installed in the two rotary tables.

The output shaft of the upper rotary table is connected with the upper drive shaft through the shaft coupling. The step motor of the upper rotary table connected with control circuit, drives the solar 
panels through driving the drive part of the upper swing mechanism, and can realize 180-degree rotation in east-west direction. In the same way, the step motor of the lower rotary table connected with control circuit, drives the solar panels through driving the drive part of the lower swing mechanism, and can realize 180-degree rotation in north-south direction.

\section{Solar Tracking System Program Design.}

At present, there are three kinds of solar tracking control methods at home and abroad: sun trajectory tracking, the photoelectric tracking and the combination of the photoelectric tracking and sun trajectory tracking[4][5]. Through the analysis and comparison of the former two control methods, this paper puts forward another new control method based on the GPS and GPRS.

\section{1 the Principle of Photoelectric Tracking.}

Photoelectric tracking system generally consists of position detector, signal processing, controller and actuator. The following figure shows the block diagram of photoelectric tracking system.

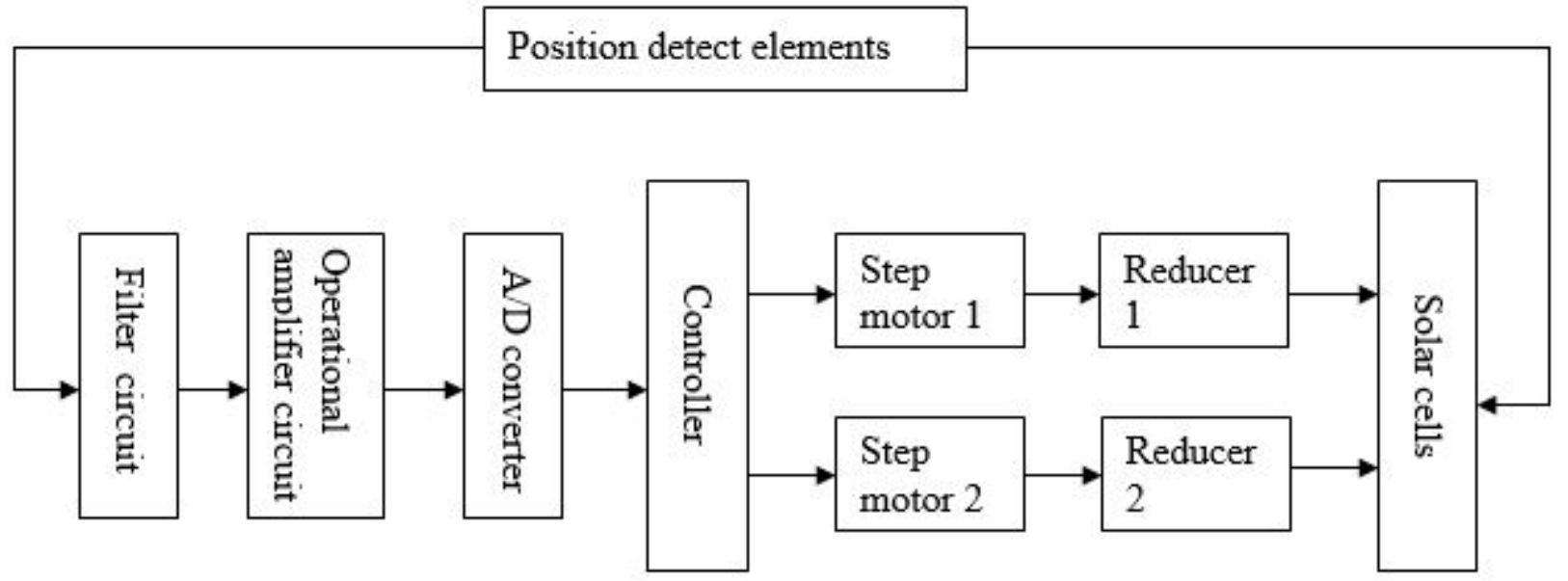

Fig. 1 the system block diagram of photoelectric tracking

Generally photoelectric tracking system adopts closed-loop control, the approach of which has a high tracking sensitivity and accuracy with its easy structure to realize. Every coin has two sides. The disadvantage of the photoelectric tracking is that system is unable to judge signals correctly, impacting the tracking results because of photosensitive element in extreme weather unable to accurately sense the intensity of the sun light.

\section{2 the Principle of Sun Trajectory Tracking.}

The principle of sun trajectory tracking is to, with the help of the computer, calculate solar elevation angle and azimuth angle at every moment from sunrise to sunset according to the sun's orbit. Then the computer transfers the sun's position calculation results to the controller. The controller commands to drive the corresponding motor rotation hereafter, and then drives the tracking device to move with the sun, realizing real-time tracking of the sun.

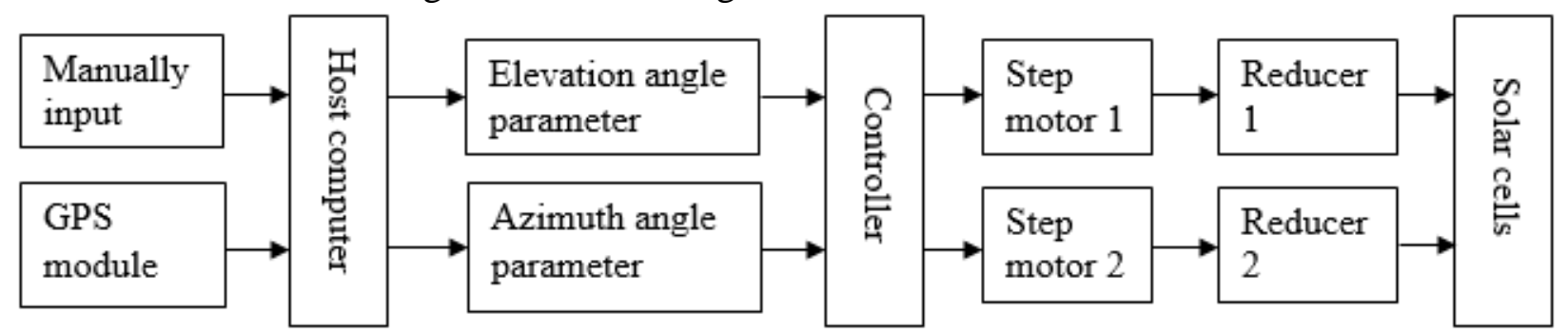

Fig. 2 the system block diagram of sun trajectory tracking

As the Fig. 2 shows, sun trajectory tracking is mainly composed of signal processing, controller and actuator. The signal processing of sun trajectory tracking mainly depends on the results of astronomical algorithms calculation[6]. Therefore, the accuracy of astronomical directly affects the accuracy of the calculated results, leaving that the most obvious defects of sun trajectory tracking. Hence algorithm must be studied particularly. 


\section{3 the Improved Sun Trajectory Tracking Based on the GPRS.}

Photoelectric tracking is unable to control rotated angle on a cloudy day, causing a low photoelectric transformation efficiency. Besides light sensor has been in a state of work, generating a certain power consumption. Research shows that the sun trajectory tracking is the ideal tracking strategy. [52] Though sun trajectory tracking can stably control the angle of panels on a cloudy day, it can not receive any signal about the extreme weather. If not to close it artificially, the panels will not only generate a certain consumption, but also will be somewhat impaired by the bad weather. From what has be presented above, the two programme both have unavoidable deficiencies. Thus, this paper put forward a new sun trajectory tracking based on GPS and GPRS. The following figure shows the block diagram of this programme.

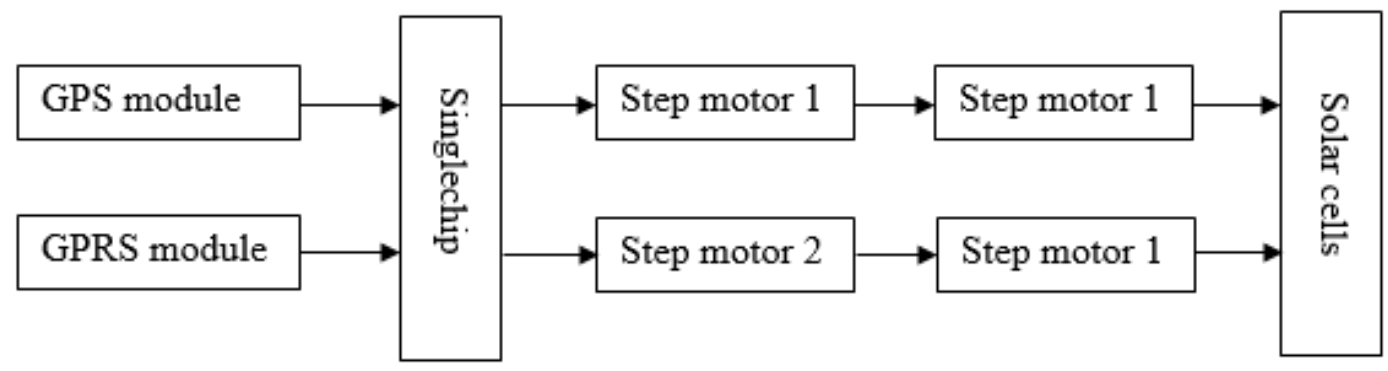

Fig. 3 the system block diagram of the improved sun trajectory tracking

The GPS and GPRS modules convey the signal to the singlechip, the singlechip calculates to determine whether the weather is extreme or not, and then the tackled result comes out, conveyed to the two step motors to adjust the angle of the solar panels. If weather is so bad that there is no point in holding on working, the microprocessor will stop the two motors. If the weathre are not so bad, the microprocessor will continue controlling the two motors to rotate the panels. At the same time, with the help of webservice, manager of the solar power station can acquainted with the operation of the power station at any time. Therefore, if there is a panels can not work, the manager will send people to repair timely.

Compared with the former two programmes, this way shows great advantages in avoiding meaningless power consumption and tackling sudden events. The schematic diagram of the advanced tracking system is shown below.

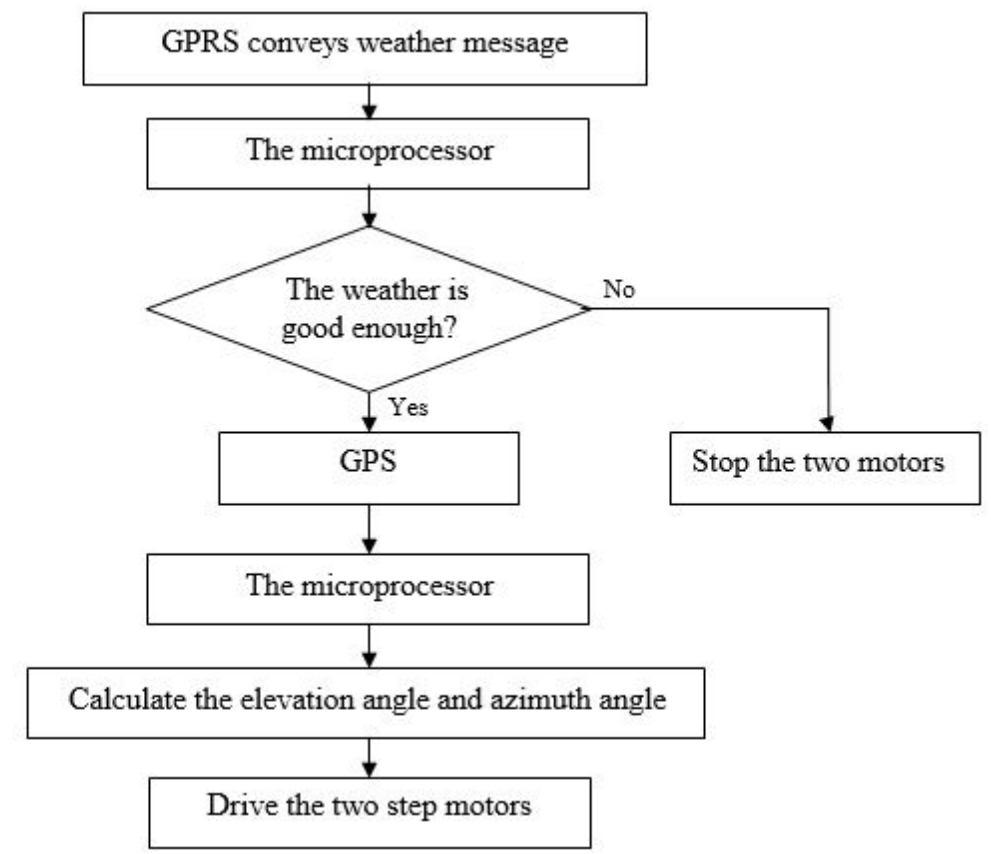

Fig. 4 the schematic diagram of the advanced tracking system 


\section{Hardware Circuit of the Control System Design.}

The system mainly includes information collection module, controller, actuator module, solar panels, solar charging controller, storage batteries and power module. Signal collection module consists of GPS module, GPRS module and A/D converter. The signals from the GPS and GPRS modules will connect with $\mathrm{A} / \mathrm{D}$ converter. $\mathrm{A} / \mathrm{D}$ converter is connected with controller by serial port. Actuator is composed of drive circuits, step motors and mechanical device. The specific circuit is shown below partly.

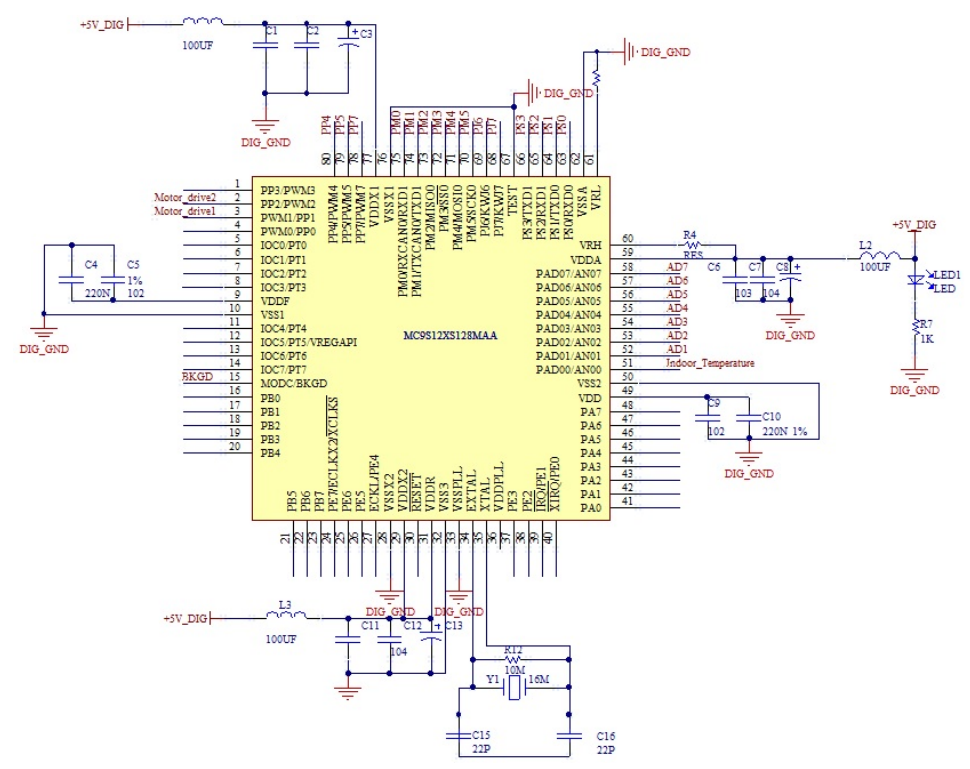

Fig. 5 the circuit design of the advanced tracking

\section{Summary}

This paper designs a dual axis mechanism, and put forward a solar tracking scheme based on the GPS and GPRS. If the weather is good enough, the control is the same with the sun trajectory tracking. If the weather is not good enough, the microprocessor will stop the work of the two step motors. Though the design is creative to a certain degree, because of limited person capacity and time, the design of the hardware mechanism and circuit also have some deficiencies. In actual application, there are still many questions need to be tackled.

\section{References}

[1]. Henrik Lund. Renewable energy strategies for sustainable development[M].Energy,2007, 32(6):912-919.

[2]. Tomson T. discrete two-positional tracking of solar collectors[J]. Renewable energy, 2008, 33(3):400-405.

[3]. GST-520 biaxial solar tracking sensor introduction. Shenzhen Sigu technology Co. Ltd.2011.(China)

[4]. Alistair B. Sproul. Derivation of the solar geometric relationships using vector analysis[J]. Renewable Energy. 2007 (32):1187-1195.

[5]. Bashir Ahmad. Users and disusers of box solar cookers in urban India-Implications for cooking projects. Solar Energy. 2000，69: 209-215.

[6]. Reda I, Andreas A. Solar position algorithm for solar radiation applications[J]. solar energy, 2004, 76(5):577-589. 